\title{
Hippocampal and Insular Response to Smoking-Related Environments: Neuroimaging Evidence for Drug-Context Effects in Nicotine Dependence
}

\author{
F Joseph McClernon*, I,2, Cynthia A Conklin³, Rachel V Kozink', R Alison Adcock ${ }^{1,4}$, Maggie M Sweitzer', \\ Merideth A Addicott' ${ }^{1,2}$, Ying-hui Chou ${ }^{1,2}$, Nan-kuei Chen ${ }^{2,5}$, Matthew B Hallyburton' and \\ Anthony M DeVito'
}

'Department of Psychiatry and Behavioral Sciences, Duke University School of Medicine, Durham, NC, USA; ${ }^{2}$ Duke-UNC Brain Imaging and Analysis Center, Duke University School of Medicine, Durham, NC, USA; ${ }^{3}$ Department of Psychiatry, University of Pittsburgh Medical Center, Pittsburgh, PA, USA; ${ }^{4}$ Center for Cognitive Neuroscience, Duke University, Durham, NC, USA; ${ }^{5}$ Department of Radiology, Duke University School of Medicine, Durham, NC, USA

\begin{abstract}
Environments associated with prior drug use provoke craving and drug taking, and set the stage for lapse/relapse. Although the neurobehavioral bases of environment-induced drug taking have been investigated with animal models, the influence of drugenvironments on brain function and behavior in clinical populations of substance users is largely unexplored. Adult smokers $(n=40)$ photographed locations personally associated with smoking (personal smoking environments; PSEs) or personal nonsmoking environment (PNEs). Following 24-h abstinence, participants underwent $\mathrm{MRRI}$ scanning while viewing PSEs, PNEs, standard smoking and nonsmoking environments, as well as proximal smoking (eg, lit cigarette) and nonsmoking (eg, pencil) cues. Finally, in two separate sessions following 6-h abstinence they viewed either PSEs or PNEs while cue-induced self-reported craving and smoking behavior were assessed. Viewing PSEs increased blood oxygen level-dependent signal in right posterior hippocampus ( $\mathrm{PHPC} ; F_{2,685}=3.74, p<0.024$ ) and bilateral insula (left: $F_{2,685}=6.87, p=0.00 \mathrm{I}$; ; right: $F_{2,685}=5.34, p=0.005$ ). In the laboratory, viewing PSEs, compared with PNEs, was associated with higher craving levels $\left(F_{2,180}=18.32, p<0.000 \mathrm{I}\right)$ and greater ad lib smoking $\left(F_{1,36}=5.0 \mathrm{I}, p=0.032\right)$. The effect of PSEs (minus PNEs) on brain activation in right insula was positively correlated with the effect of PSEs (minus PNEs) on number of puffs taken from a cigarette $(r=0.6, p=0.001)$. Our data, for the first time in humans, elucidates the neural mechanisms that mediate the effects of real-world drug-associated environments on drug taking behavior under conditions of drug abstinence. These findings establish targets for the development and evaluation of treatments seeking to reduce environment provoked relapse.

Neuropsychopharmacology (2016) 4I, 877-885; doi:I0.1038/npp.20I5.2 I4; published online 28 October 2015
\end{abstract}

\section{INTRODUCTION}

Environments associated with prior drug use increase craving and provoke relapse in addicts (O'Brien et al, 1992). Clinicians and recovery programs have long recognized the powerful influence of environmental contexts and often counsel drug users to avoid or learn to effectively cope with being in places associated with past drug use (Stalcup et al, 2006). Although extensive animal research has investigated the neurobiology of preference for, and reinstatement of drug seeking in, drug-associated environments (Fuchs et al, 2008; Marchant et al, 2014), very little is known about the neural substrates of exposure to drug-related environments in humans. Such an understanding is critical in developing

\footnotetext{
*Correspondence: Dr FJ McClemon, Duke University School of Medicine, Box 3527, Durham, NC 277I0, USA, Tel: +I 919668 3987, Fax: + 9196810016 , E-mail: francis.mcclernon@duke.edu Received I4 May 20I5; revised 12 June 20I5; accepted I July 20 I5; accepted article preview online 16 July 2015
}

and evaluating novel interventions that specifically target relapse triggered by drug environments in clinical populations.

The neural substrates underlying the learning and retrieval of drug-environment associations have been investigated with animal models in which environments are paired with drug effects (eg, conditioned place preference; CPP; (Aguilar et al, 2009; Liu et al, 2008; Napier et al, 2013)) or reinforcement (eg, context-induced reinstatement; CIR; (Fuchs et al, 2008; Marchant et al, 2014)). Neuropharmacological studies of drug-environment associations using these paradigms implicate brain regions broadly involved in conditioned reward including the amygdala, medial prefrontal cortex (mPFC), striatum, and insula (Bossert et al, 2011; Fuchs et al, 2005; McLaughlin and See, 2003; Otis et al, 2014). However, one region - the dorsal hippocampus (dHPC) - has been singled out as having a potentially selective and critical role in drugcontext learning (Fuchs et al, 2005; Meyers et al, 2003, 2006; Ramirez et al, 2009). In an earlier study (Meyers et al, 2003), inactivation of the dorsal but not ventral HPC attenuated 
cocaine CPP. In another study, Fuchs et al (2005) found that dHPC inactivation attenuated contextual reinstatement of drug seeking, but did not attenuate reinstatement in response to an explicit conditioned stimuli (CSs) or to a priming dose of drug. These and other studies of context/environmentreward conditioning (Meyers et al, 2006) are consistent with hypothesized functional specialization of the dHPC (analogous to posterior HPC (pHPC) in humans) in forming and retrieving fine-grained representations of the environment bound to salient motivational stimuli (eg, rewards) (Poppenk et al, 2013; Strange et al, 2014; Wolosin et al, 2012).

The present study of human smokers was designed to extend prior animal research by evaluating the effects of exposure to smoking-related environments on activation in HPC and other brain areas involved in conditioned reward (ie, amygdala, mPFC, insula, striatum), and to examine correlations between brain reactivity, cue-provoked craving, and smoking behavior. We employed a previously validated paradigm (Conklin et al, 2010), in which smokers are exposed in the laboratory to their personally generated pictures of the places, in which they typically smoke or refrain from smoking. In prior research we found that exposure to personal smoking environments (PSEs) evoked greater cueinduced craving compared with personal nonsmoking environments (PNEs), as well as standard (non-personal) smoking and nonsmoking environments (Conklin et al, 2010). In the present study, we hypothesized that viewing smoking-related cues would increase blood oxygen leveldependent (BOLD) signal in conditioned reward areas, as identified in prior neuroimaging studies (Kuhn and Gallinat, 2011), but that PSEs would selectively increase BOLD signal in $\mathrm{pHPC}-\mathrm{a}$ region analogous to the dHPC in rodents.

\section{MATERIALS AND METHODS}

\section{Overview of Study Design}

In the study, participants completed five sessions in the following order: (1) a screening session in which inclusion/ exclusion criteria were evaluated; (2) a training session in which participants learned about the picture taking protocol and underwent a mock fMRI session; (3) a brief camera dropoff visit; (4) a cue-exposure fMRI session following 24-h abstinence to assess neural reactivity to all cues; and (5 and 6) two cue-exposure sessions conducted outside of the scanner during which self-report craving and behavioral reactivity (eg, number of puffs) to personal smoking and nonsmoking environment cues were assessed in separate sessions.

\section{Participants}

Forty smokers between the ages of $18-55$ years were recruited from the community and completed the fMRI session of the study. Inclusion criteria for all subjects included being right handed, free of serious health problems, not currently using psychoactive medications, not having any conditions making MRI research unsafe, testing negative for illicit drug use, and among females, having a negative pregnancy test. Participants were required to smoke $\geqslant 5$ cigarettes/day for $>1$ year, have an expired $\mathrm{CO}$ concentration of $\geqslant 9$ p.p.m. or a positive urine cotinine test of $\geqslant 100 \mathrm{ng} / \mathrm{ml}$ ( $\geqslant 3$ on Nicalert urine test strip), no current use of any nicotine products other than cigarettes and report no immediate interest in quitting. All participants read and signed an IRB-approved informed consent form.

\section{Picture Taking Protocol}

After meeting all study inclusion/exclusion criteria, participants were trained to take pictures of personal smoking (PSEs) and nonsmoking (PNEs) environments. Using methods validated in a previous study (Conklin et al, 2010), each participant was interviewed to determine four to five specific environments in which they frequently smoke (ie, smoke at least 7 out of 10 times they are there) or refrain (smoke $<3$ out of 10 times there). They were then given a digital camera and trained to acquire four pictures of each environment, two approaching and two within (see Supplementary Figure S1). Upon returning the camera, study staff reviewed all pictures to determine picture content and quality. As needed, pictures were photo edited to remove proximal smoking cues (eg, ashtrays, packs of cigarettes) and any people.

\section{fMRI Session and Task}

As in prior research (McClernon et al, 2009) and to maximize brain reactivity, participants were required to be 24-h abstinent at the start of the fMRI session as confirmed by breath carbon monoxide level $\leqslant 6$ p.p.m. fMRI task stimuli (Figure 1) were the personal pictures acquired by each participant, a set of standard smoking and nonsmoking environments from a previously validated set (Conklin et al, 2008, 2010) and proximal cues depicting smoking (eg, cigarette, ashtray) and nonsmoking (eg, pencil, pad) objects. Pictures were presented in the scanner in a block design format (block length $=16 \mathrm{~s}$; four pictures per block). Stimulus duration within each block varied from 3-5 s. After each block, participants were asked to rate their craving on a scale from 1 (not at all) to 8 (extremely) with a bimanual response pad. Inter-block intervals were $16 \mathrm{~s}$ in length. A total of 60 blocks (10 per category) were presented across 4, 8.3 min runs. Each stimulus was presented twice and the order of blocks and runs was counterbalanced.

\section{fMRI Acquisition, Preprocessing and First Level Modeling}

See the Supplementary Information for additional fMRI acquisition detail. BOLD images were acquired using gradient-recalled inward spiral pulse imaging with $\mathrm{K}$-space trajectory on a $3 \mathrm{~T}$ scanner. BOLD signal underwent preprocessing in FSL version 5.0.1 (Jenkinson et al, 2012): (1) rigid-body motion correction using MCFLIRT (Jenkinson et al, 2002); (2) slice-timing correction; (3) spatial smoothing using an $8 \mathrm{~mm}$ FWHM Gaussian kernel; (4) high-pass filtering; and (5) registration to standard space using FLIRT. Each block was modeled as a boxcar function with duration of $16 \mathrm{~s}$ convolved with double $\gamma$ hemodynamic response function.

\section{fMRI Data Analysis}

Region of interest (ROI) analysis. In view of the functional heterogeneity of the HPC along the anterior-posterior axis (Poppenk et al, 2013; Strange et al, 2014), separate hippocampal region of interests (ROIs) were generated for 

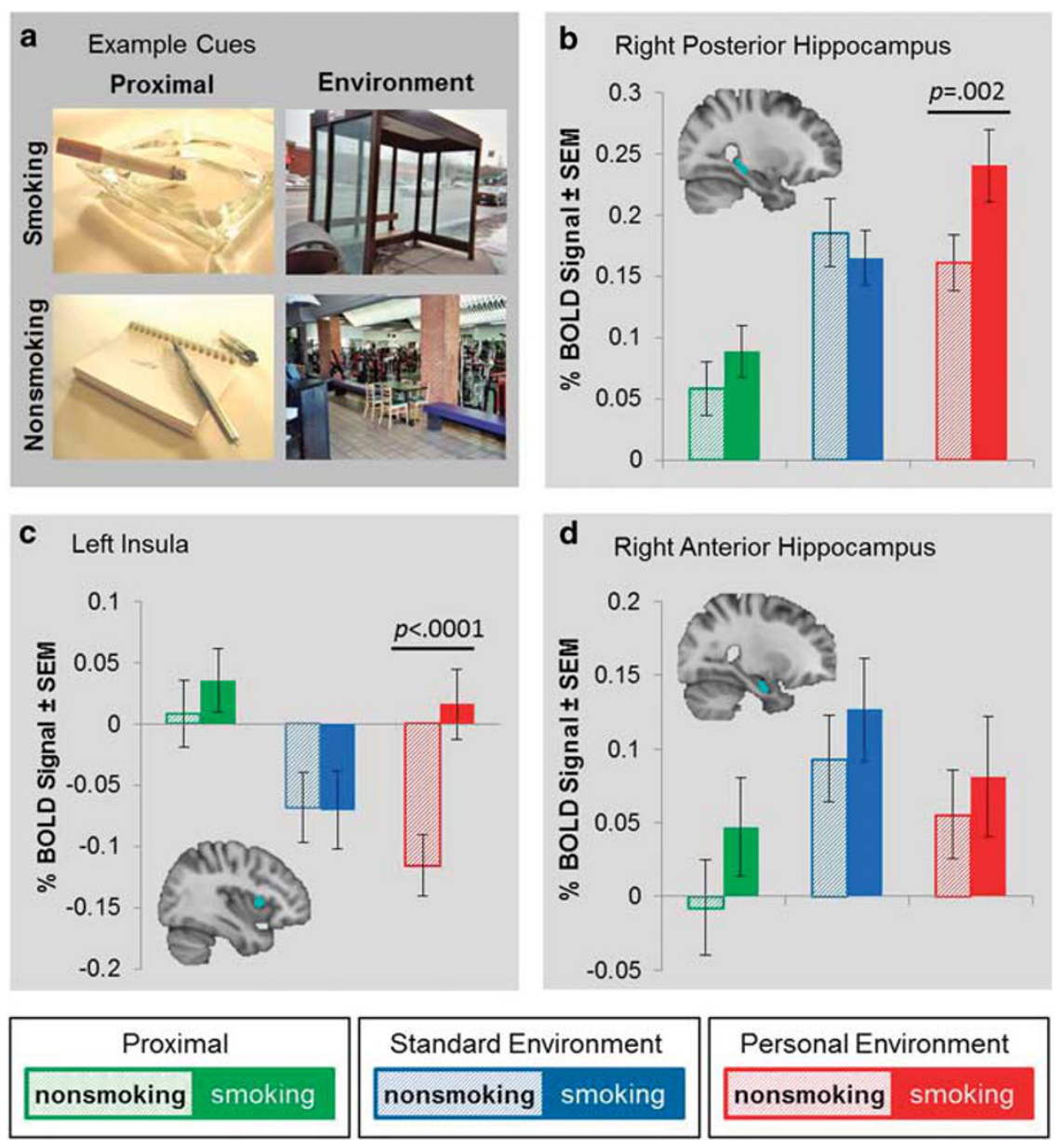

Figure I Example cues and differential effects of viewing personal and standard smoking and nonsmoking environments on brain activation-regions of interest (ROI) analysis. (a) Depiction of samples of smoking and nonsmoking, proximal and environment cues. (b-d) Bar graphs represent mean \% BOLD signal change in response to viewing smoking and nonsmoking cues for each category in right posterior and anterior hippocampus ( $\mathrm{pHPC}$ and aHPC in Panels b and d, respectively) and left insula (INS; (c)). Error bars represent SEM. Viewing personal smoking environments (PSEs) was associated with selectively increased BOLD signal in right pHPC and bilateral INS (see text and Supplementary Figure S3 for additional statistical detail).

anterior and posterior HPC. Bilateral aHPC and pHPC ROIs were defined following a prior study of aHPC and pHPC connectivity (Chen and Etkin, 2013), taking the anterior-most third and posterior-most third of an HPC ROI consisting of voxels with a $>60 \%$ likelihood of being in HPC (as defined by the Harvard-Oxford probabilistic structural axis). The following additional ROIs, chosen a priori based on preclinical research (Bossert et al, 2011; Fuchs et al, 2005; McLaughlin and See, 2003; Otis et al, 2014), our previous neuroimaging studies (McClernon et al, 2005, 2007, 2008, 2009) and metaanalysis (Engelmann et al, 2012; Kuhn and Gallinat, 2011; Tang et al, 2012) of cue-reactivity, were created in PickAtlas (Maldjian et al, 2003): (1) ventral striatum (5 $\mathrm{mm}$ radius sphere centered on $\pm 6,4,-5$ ); (2) amygdala (anatomical); (3) insula ( $5 \mathrm{~mm}$ radius sphere centered on $\pm 38,10,6)$; (4) $\mathrm{mPFC}$ inclusive of portions of rostral anterior cingulate cortex $(5 \times 10 \times 10 \mathrm{~mm}$ box centered on $\pm 5,40,10)$; and (5) PCC $(5 \times 5 \times 10 \mathrm{~mm}$ box centered on $\pm 5,-60,20)$. See Supplementary Figure S3 for visual depictions of the ROIs selected for analyses. Mean percent BOLD signal change values were extracted for each block type and ROI and submitted to a general linear mixed model (GLMM) with subject-level Gaussian random effects, and with CATEGORY (personal environment, standard environment, proximal) and CUE (smoking, nonsmoking) as fixed factors. Given the specificity of our HPC hypotheses, tests involving those ROIs were evaluated at $\alpha=0.05$; tests involving all other ROIs were evaluated at $\alpha=(0.05 / 10$ ROIs $)=0.005$.

Exploratory whole-brain analysis. In addition to ROIbased analysis, three exploratory whole-brain analyses were conducted to identify non-hypothesized effects (ie, reduce risk of Type II error) and contribute data to future meta-analyses. First, we examined activation that was greater in response to PSEs compared with PNEs. A second analysis was conducted to identify cue-provoked brain activation that was greater in response to viewing PSEs compared with standard smoking environments minus any effects of viewing nonsmoking environments: (personal environments (smoking $>$ nonsmoking) $>$ standard environments (smoking $>$ nonsmoking)). Finally, we searched for activation that was greater in response to viewing any smoking environments compared with proximal smoking cues (personal or standard environments (smoking $>$ nonsmoking) $>$ proximal (smoking $>$ nonsmoking)). A cluster-corrected threshold of $z=3.1, p<0.05$ was employed. 
Functional connectivity analysis. To identify networks of brain regions associated with PSE reactivity and smoking behavior, an exploratory probabilistic independent component analysis on BOLD signal acquired during the task was conducted in FSL MELODIC (see Supplementary Information for details). A single component that included both hippocampal and insula ROIs was identified and correlations between intrinsic connectivity in these ROIs and the effects of PSEs on smoking behavior were assessed.

\section{Cue-Exposure Sessions}

The behavioral and subjective effects of viewing the personal environment cues were assessed within 1 month of the fMRI session in two separate laboratory sessions-one using PSEs; the other using PNEs (order randomly assigned and counterbalanced; occurring a median of 7 and 14 days post-fMRI session). In order to minimize ceiling effects on self-reported craving and as in a prior study, participants were required to be only 6 -h abstinent at the start of each session as indicated by a breath $\mathrm{CO}$ level that was $60 \%$ of their screening CO level (Conklin et al, 2010). Following assessment of baseline breath $\mathrm{CO}$ and craving, participants were seated in front of a 21-inch computer monitor. They were instructed to focus intently on the environments displayed on the screen. Following an initial 2-min exposure to cues, after which cue-induced craving was assessed, participants viewed cues for an additional $12 \mathrm{~min}$ during which they were given ad libitum access to their cigarettes and were informed that they could smoke freely. During this ad lib smoking period, participant behavior was video recorded and the following variables later coded by raters: no. of cigarettes smoked, latency to first puff and number of puffs. Following the ad lib period, cueprovoked craving and breath $\mathrm{CO}$ levels were assessed a final time. Data were entered into GLMM with subject-level Gaussian random effects; a negative binomial distribution was assumed for count data.

\section{RESULTS}

\section{Participant Characteristics}

Participants in the full sample ( $n=40$ ) were 59\% female, $64 \%$ nonwhite, 35 years of age $(S D=11.1)$, and smoked 14.8 $(\mathrm{SD}=6.5) \quad$ cigarettes/day. Complete and usable data sets were available for $n=37$ and $n=30$ participants for laboratory and neuroimaging outcomes, respectively (see Supplementary Information for additional screening and demographic information).

\section{BOLD Signal and Subjective Reactivity to Personal Smoking Environments}

In-scanner craving ratings. As shown in Supplementary Figure S2 and consistent with our prior research (Conklin et al, 2010), PSEs elicited greater craving than PNEs ( $t=13.5$, $p<0.0001)$. Although craving was greater in response to smoking compared with nonsmoking stimuli for both standard environments $(t=10.2, p<0.0001)$ and proximal cues $(t=8.76, p<0.001)$, cue-provoked craving (ie, craving in response to smoking versus nonsmoking stimuli) was greater for personal environments than standard environments $(t=2.74, p=0.008)$ and similar to that observed for proximal cues $(t=-1.23, p=0.224)$.

Hippocampal ROIs. As can be seen in Figure 1 and Supplementary Figure S3, in each of the HPC ROIs, there was a main effect of CUE, with greater signal in response to viewing smoking versus nonsmoking stimuli $\left(F^{\prime} s_{1,685}>3.94\right.$, $p$ 's $<0.05)$. We also observed significant main effects of CATEGORY in each ROI $\left(F^{\prime} s_{1,685}>4.0, p\right.$ 's $\left.<0.05\right)$ generally due to environments (whether personal or standard) eliciting greater activation than proximal cues. Finally, as shown in Figure 1, in the right pHPC, we found a significant CATEGORY $X$ CUE interaction, $F_{2,685}=3.74, p<0.024$ such that BOLD signal was greater in response to smoking as compared with nonsmoking cues only for personal $(p=0.002)$, but not standard environments $(p=0.43)$ or proximal cues $(p=0.24)$.

Other ROIs. As can be seen in Supplementary Figure S3, main effects of CATEGORY $\left(F^{\prime} s_{2,685}>8.7, p\right.$ 's $\left.<0.001\right)$, reflecting greater BOLD activation in response to personal environment cues, were observed in right vSTR, and in bilateral INS, mPFC, and PC, but not AMG or left vSTR. Main effects of CUE $\left(F^{\prime} s_{1,685}>8.27, p ' s<0.005\right)$, reflecting greater BOLD activation in response to all smoking compared with all nonsmoking stimuli, were observed in three left hemisphere ROIs-INS, AMG, and mPFC. Finally, as can be seen in Figure 1 and Supplementary Figure S3, significant CATEGORY x CUE interactions were observed in left $\left(F_{2,685}=6.87\right.$, $p=0.0011)$ and right $\left(F_{2,685}=5.34, p=0.005\right)$ INS. In these regions, planned comparisons indicated that there was less deactivation in response to viewing PSEs as compared with PNEs (left: $p<0.0001$, right: $p=0.001$ ). Effect sizes associated with the difference in reactivity between smoking and nonsmoking cues for each category are presented in Supplementary Table S3.

Exploratory whole-brain analysis. As shown in Figure 2 and Supplementary Table 2, viewing PSEs, relative to PNEs, elicited significant BOLD activation in brain areas including bilateral parietal cortex, right temporal gyrus, right insula, right striatum, right supramarginal gyrus, cerebellum, and brainstem. No regions were more activated for PNEs compared with PSEs. BOLD signal was also greater in response to viewing personal smoking compared with standard smoking environments, controlling for effects of nonsmoking environments, in one large cluster encompassing the left insula and putamen. As can be seen in Figure 2, this cluster overlapped considerably with the a priori insula ROI. Finally, BOLD signal was greater in response to viewing smoking environments (standard or personal) relative to proximal smoking cues, controlling for all nonsmoking stimuli, in bilateral lateral occipital and right temporal areas and right precuneus.

\section{Effects of Personal Environments on Craving and Behavior}

As shown in Figure 3, during the cue-exposure sessions, exposure to PSEs relative to PNEs resulted in greater craving $\left(F_{2,180}=18.32, p<0.0001\right)$. Smokers reported significantly 


\section{a $P S E>P N E$}

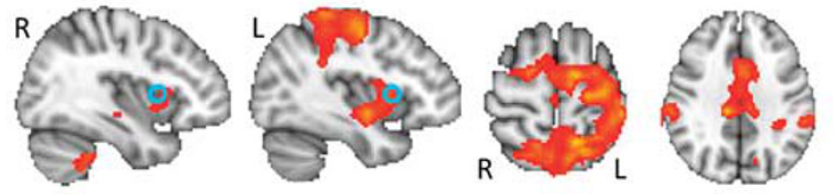

b Personal $(\mathrm{S}>\mathrm{N})>$ Standard $(\mathrm{S}>\mathrm{N})$

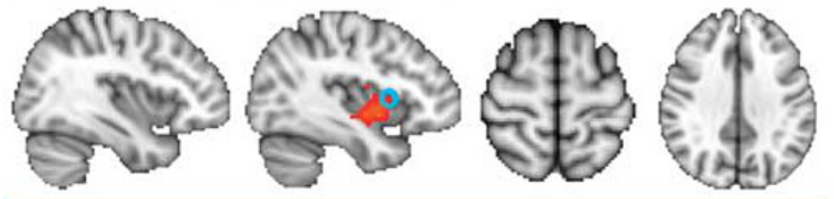

C Environment $(\mathrm{S}>\mathrm{N})>$ Proximal $(\mathrm{S}>\mathrm{N})$

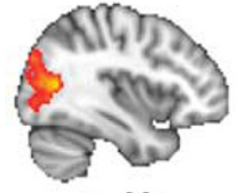

$x=38$

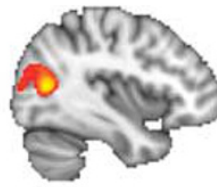

$x=-38$

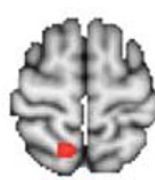

$z=62$

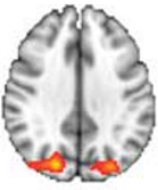

$z=34$ $z=3.1=5$

Figure 2 Differential effects of viewing personal and standard smoking and nonsmoking environments on brain activation - whole-brain, voxel-wise analysis. Selected whole-brain, voxel-wise analyses were carried out. (a) BOLD signal was greater in response to viewing personal smoking environments, compared with personal nonsmoking environments (PSE > PNE) in brain areas including bilateral parietal cortex, right temporal gyrus, right insula, right striatum, right supramarginal gyrus, cerebellum, and brainstem. (b) Activation in response to smoking compared with nonsmoking cues was greater for personal compared with standard environments (personal > standard) in left insula. (c) Activation in response to smoking environments (relative to nonsmoking environments) compared with proximal smoking cues (relative to nonsmoking cues; environments > proximal) was observed in bilateral lateral occipital cortex, right temporal cortex, and right precuneus. All contrasts were evaluated at a clustercorrected threshold of $z=3.1, p<0.05$. The location of the a priori INS ROls are noted in Panels $a$ and $b$ by a light blue circle.

greater craving while viewing PSEs compared with PNEs in the initial $(t=6.84, p<0.0001)$ and ad lib smoking/ viewing period $(t=4.8, p<0.0001)$. PSEs, relative to PNEs, also resulted in more puffs taken from the first cigarette $\left(F_{1,36}=5.01, p=0.032\right)$, as well as all cigarettes $\left(F_{1,36}=4.37\right.$, $p=0.044)$ smoked during the ad lib period. Latency to first puff did not differ between conditions. Finally, CO boost (post-session $\mathrm{CO}-$ pre-session $\mathrm{CO}$ ) was greater in the PSE compared with PNE condition $\left(F_{1,31}=6.65, p=0.015\right)$.

\section{BOLD Signal-Behavior Correlations}

Relations were evaluated between the effects of PSEs (minus PNEs) on smoking behavior (number of puffs from first cigarette smoked) and BOLD signal in response to PSEs (minus PNEs) in a priori pHPC and INS ROIs, while controlling for nicotine dependence level as measured by the Fagerström Test for Nicotine Dependence (Heatherton et al, 1991). Change in number of puffs (PSE-PNE) was demeaned and $\log$ transformed for the purpose of this analysis; and data from one participant was removed following multivariate outlier detection (see Supplementary Information). As shown in Figure 4, a significant correlation was observed only for right INS $(r=0.6, p=0.001)$. Analyses with craving did not yield significant results in any ROI $\left(r^{\prime} s<|0.33|\right)$.

\section{Exploratory Functional Connectivity Analysis}

As shown in Figure 5, one component (IC 7; 4.01\% of total variance) was positively associated with a priori INS and pHPC ROIs, parahippocampal cortex, superior temporal gryus, lingual gyrus, and negatively associated with the dorsal striatum. Confirming the functional relevance of this network, we observed a correlation between PSE-PNE changes in number of puffs and intrinsic connectivity of this component with the left INS ROI $(r=0.41, p=0.03)$.

\section{DISCUSSION}

The results of this study provide the first evidence in humans that exposure to environments associated with past smoking behavior under conditions of abstinence increases brain signal in the posterior hippocampus-a region identified in animal studies as critically involved in the learning and retrieval of drug-environment associations. Moreover, ROIbased and whole-brain analyses implicated additional brain regions in PSE processing, including bilateral insula, parietal cortex, temporal cortex, and striatum. Finally, we observed that PSEs increased number of puffs relative to PNEs, and this increase was predicted by differential brain reactivity to these cues in right insula. Collectively, these findings shed new light on the influence of environments in drug use and have important implications for the development and validation of new treatments for nicotine dependence and other drugs of abuse.

As hypothesized, viewing environments associated with one's personal smoking history, compared with personal nonsmoking environments, resulted in greater BOLD signal in the pHPC. Similar differences in activation in response to smoking $v s$ nonsmoking cues were not observed for proximal cues or standard environments. Our findings that the pHPC is selectively involved in processing information about environmental cues for which there is a personal history of smoking are consistent with animal studies, in which inactivation of the analogous dorsal HPC has been shown to disrupt expression of psychostimulant CPP (Meyers et al, 2003, 2006) and CIR (Fuchs et al, 2005). These animal studies of learned reward are also consistent with human and animal fear conditioning studies, in which the dorsal HPC has been shown to have a central role in context effects (Alvarez et al, 2008; Bast et al, 2003; Maren et al, 2013; Marschner et al, 2008). Interestingly and in contrast with pHPC, aHPC exhibited greater signal in response to smoking, relative to nonsmoking, cues regardless of cue category. Collectively, our findings for anterior and posterior HPC are consistent with conceptualizations of functional specialization of the anterior-posterior axis of the HPC (Poppenk et al, 2013; Strange et al, 2014) in which aHPC produces global, gist-like representations (eg, reward $v s$ neutral) and pHPC produces fine-grained, local representations (eg, the specific location of a reward in the environment). Finally, it is worth noting that hippocampus has rarely been identified in prior neuroimaging studies of 
Personal Environment

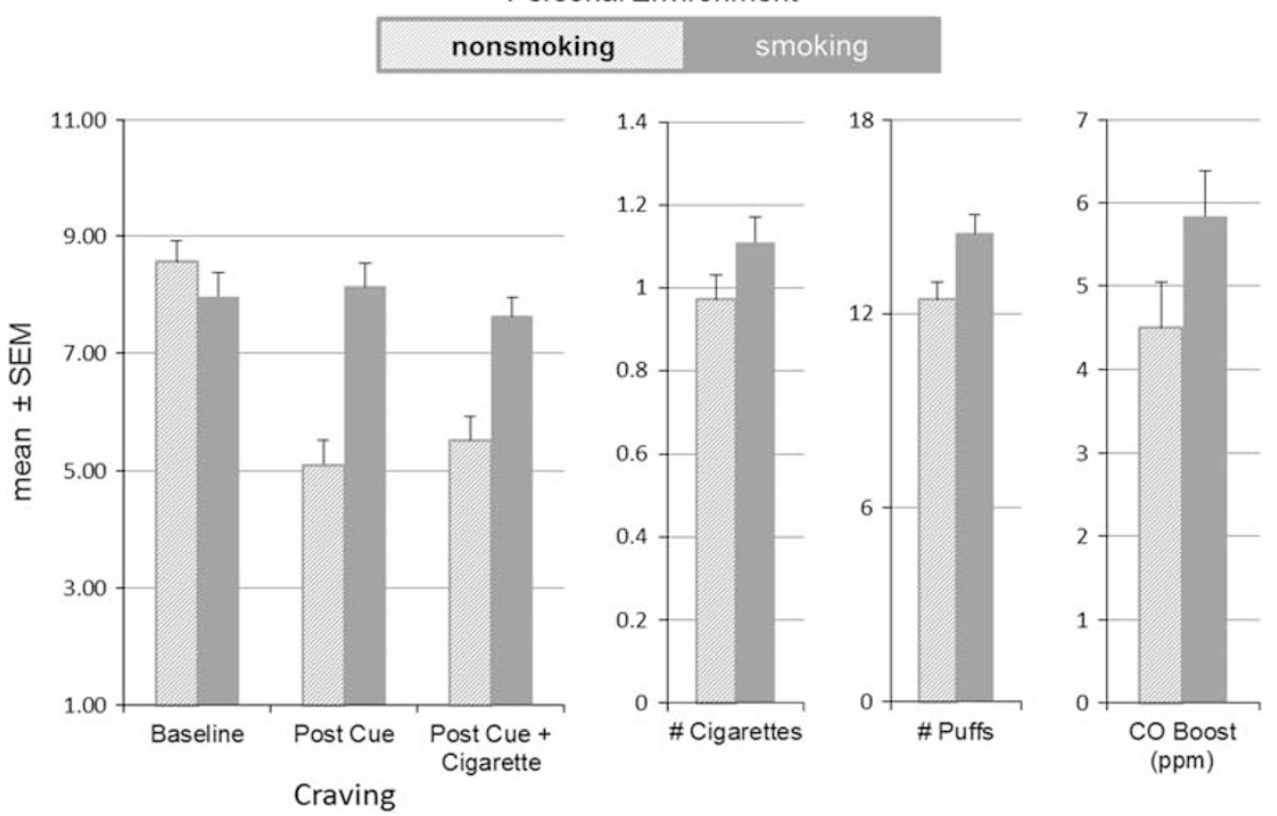

Figure 3 Differential effects of viewing personal smoking and nonsmoking environments on craving, smoking behavior and breath carbon monoxide. The effects of viewing personal smoking and nonsmoking environments are shown for craving, smoking behavior measures (no. of cigarettes smoked; no. of puffs) and boost in breath CO levels. Viewing personal smoking compared with nonsmoking environment cues (PSE vs PNE) resulted in significantly greater cueprovoked craving, higher number of puffs taken, and an increase in breath $\mathrm{CO}$ boost.

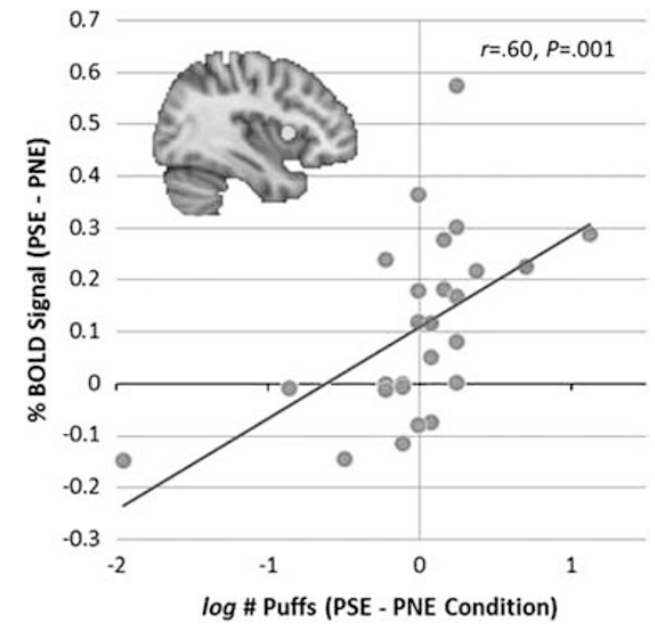

Figure 4 Association between task-related insula activation and the effects of personal smoking environments on smoking behavior. Shown is a positive association between the effects of exposure to personal smoking environments (minus nonsmoking environments) on right insula (INS) activation and on log-transformed number of puffs. This effect was observed after controlling for severity of nicotine dependence (as measured by the FTND) $(r=0.60, p=0.00 \mathrm{I})$. Although not identified as an outlier, the removal of one participant with a relatively large decrease in smoking reduces the correlation to $r=0.54, p=0.005$, two-tailed.

cue-reactivity (Wetherill et al, 2013) possibly because studies have largely used proximal or non-environment cues.

Less expected were differential effects of PSEs on brain activation in INS. In both left and right INS, PSEs resulted in less deactivation than PNEs, an effect that was not observed for standard environments and proximal cues. The INS has been proposed as a critical substrate of tobacco use: smokers with insula damage exhibit increased likelihood of quitting smoking (Gaznick et al, 2014; Naqvi et al, 2007); selfreported craving (Kuhn and Gallinat, 2011; Tang et al, 2012) and cessation outcomes (Janes et al, 2010) are correlated with insula reactivity to proximal smoking cues; and inactivation of insula decreases proximal cue-provoked nicotine seeking in rodents (Forget et al, 2010; Pushparaj et al, 2013). Less is known about the role of the insula in contextual drug effects; however, in a recent study, insula inactivation in rats led to disruption of amphetamine CPP (Contreras et al, 2012).

We also observed that right INS activation in response to PSEs (minus PNEs) was correlated with the number of puffs smokers took while viewing PSEs (minus PNEs). Interestingly, the a priori ROI in which this effect was observed was based on a meta-analysis which found high correlations between cue-provoked craving and brain reactivity to smoking cues in right dorsal anterior INS (Kuhn and Gallinat, 2011). Collectively, these data, along with activation of INS by PSEs and correlation between INS intrinsic effective connectivity with PSE-induced smoking behavior, provide strong support for a role of the INS in supporting drug-taking behavior provoked by exposure to drug environments. To our knowledge, these findings are the first direct demonstration of a link between cue-provoked changes in brain function and cue-provoked changes in smoking behavior.

Conceptualizations of the INS in addiction focus on its role in interoception and motivation, and the anterior insula (the location of our ROI) specifically has been proposed as a site for integrating external information about drug availability with affective and interoceptive representations of drug effects 


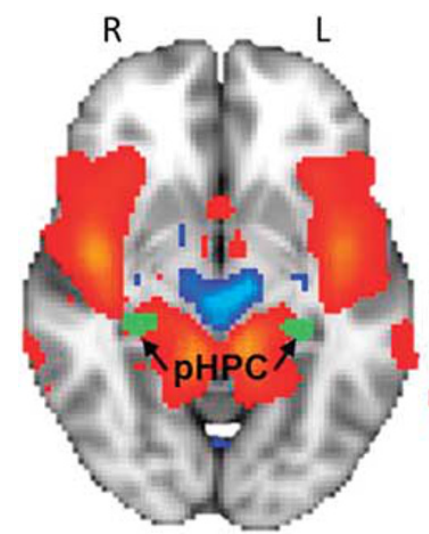

$z=-8$

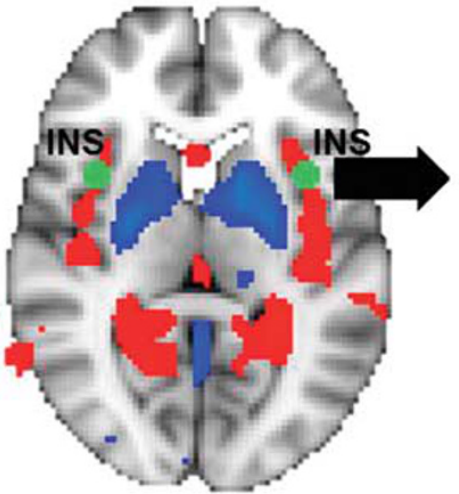

$z=8$

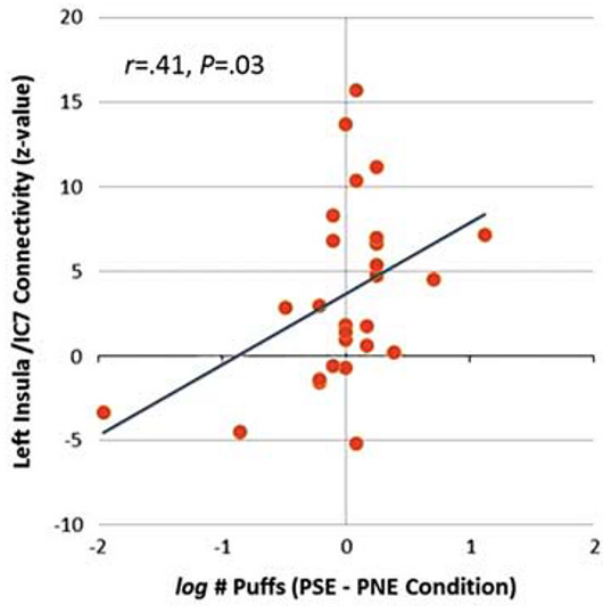

$-2.3 \quad 2.3$

19.2

Figure 5 Functional Network Including the PHPC and INS ROIs. An exploratory dual regression analysis on BOLD signal acquired during the task resulted in one component (IC 7; $4.01 \%$ of total variance) that included the a priori INS and pHPC ROls. Areas in red were positively associated with the component and include hippocampus, insula, parahippocampal cortex, superior temporal gyrus, and lingual gyrus; blue areas were negatively associated with the component and include the dorsal striatum. A priori INS and pHPC ROIs are depicted in green. A correlation between PSE-induced increases in number of puffs and intrinsic connectivity of this component with the left INS ROI $(r=0.4 \mathrm{I}, p=0.03)$ was observed. Although not identified as an outlier, the removal of one participant with a relatively large decrease in smoking reduces the correlation to a trend $(r=0.34, p=0.09$, two-tailed).

to motivate and guide drug seeking behavior (Naqvi et al, 2014). Consistent with this, our current results strongly implicate PSEs in setting the stage for smoking behavior and point to the insula as a key determinant of their behavioral impact. Our whole-brain exploratory analysis of activation specific to PSEs, further implicate a network of brain regions with roles in attention (parietal cortex), memory (temporal cortex), and reward (striatum) in the processing of these cues.

Strengths of the study include the integration of diverse methods, testing of hypotheses among a priori ROIs and within-subjects design. One important limitation of the study is that nonsmoking environments, whether personal or standard, represent a CS-, or negative predictor of smoking; as such, our paradigm lacks a neutral stimulus control condition. Thus, observed effects of nonsmoking environments may represent the inhibition of activation and behavior rather than a non- or neutral-response. We conducted a whole-brain exploratory analysis that did not reveal clusters of activation in response to personal nonsmoking $>$ smoking environments; however, further study of the influence of nonsmoking environments is warranted. Related to this, while we attempted to control for differences in picture quality and composition by using validated and standardized methods for identifying and photographing locations, there may have been systematic differences between smoking and nonsmoking locations (eg, physical factors such as lighting) and the availability of other reinforcers (eg, alcohol or social interaction). Although such variability represents natural differences in the smoker's environment and thus increases the ecological validity of the study, and whereas we attempted to control for such factors by inclusion of standard smoking and nonsmoking environments, further analysis of these issues is warranted. Moreover, we did not manipulate personalization of proximal cues. Although such stimuli would presumably be less ideographic than environments, it would be worthwhile in future studies to examine this question. Other limitations include a non-treatment seeking sample (which limits generalizability to clinical samples of smokers interested in quitting), testing that was conducted only during abstinence which might limit generalizability to other drug states including long-term abstinence, and a narrow focus on context as the physical environment, to the exclusion of both internal (eg, withdrawal, stress) and other external (eg, social) contexts.

\section{Implications and Summary}

Animal models have provided a wealth of data on the behavioral and neuropharmacological bases of drugenvironment effects. The current study validates a paradigm for studying these effects in clinical samples by demonstrating parallel neural circuitry and behavioral responding. As such, our human-subjects paradigm can be used to screen compounds effective in attenuating contextual reinstatement in animals (Napier et al, 2013). In addition, our data demonstrate that environmental cues elicit brain activation that differs in important ways from proximal cues, suggesting these cues may be critical to include in therapies seeking to extinguish or dampen conditioned responding. Finally, our data provide important new evidence in a clinical sample that environments associated with prior drug use are critical factors in drug craving and use.

\section{FUNDING AND DISCLOSURE}

FJM was a Site PI on an investigator initiated grant (GRAND) from Pfizer. The other authors declare no conflicts of interest.

\section{ACKNOWLEDGMENTS}

This work was funded by grants to Drs McClernon (R21 DA033083) and Conklin (R01 DA023646) from the National 
Institutes on Drug Abuse. ClinicalTrials.gov: Environments as Smoking Cues: Imaging Brain Substrates, Developing New Treatments (CameraCue); NCT01840111.

\section{REFERENCES}

Aguilar MA, Rodriguez-Arias M, Minarro J (2009). Neurobiological mechanisms of the reinstatement of drug-conditioned place preference. Brain Res Rev 59: 253-277.

Alvarez RP, Biggs A, Chen G, Pine DS, Grillon C (2008). Contextual fear conditioning in humans: cortical-hippocampal and amygdala contributions. J Neurosci 28: 6211-6219.

Bast T, Zhang WN, Feldon J (2003). Dorsal hippocampus and classical fear conditioning to tone and context in rats: effects of local NMDA-receptor blockade and stimulation. Hippocampus 13: 657-675.

Bossert JM, Stern AL, Theberge FR, Cifani C, Koya E, Hope BT et al (2011). Ventral medial prefrontal cortex neuronal ensembles mediate context-induced relapse to heroin. Nat Neurosci 14: 420-422.

Chen AC, Etkin A (2013). Hippocampal network connectivity and activation differentiates post-traumatic stress disorder from generalized anxiety disorder. Neuropsychopharmacology 38: 1889-1898.

Conklin CA, Perkins KA, Robin N, McClernon FJ, Salkeld RP (2010). Bringing the real world into the laboratory: personal smoking and nonsmoking environments. Drug Alcohol Depend 111: 58-63.

Conklin CA, Robin N, Perkins KA, Salkeld RP, McClernon FJ (2008). Proximal versus distal cues to smoke: the effects of environments on smokers' cue-reactivity. Exp Clin Psychopharmacol 16: 207-214.

Contreras M, Billeke P, Vicencio S, Madrid C, Perdomo G, Gonzalez $\mathrm{M}$ et al (2012). A role for the insular cortex in longterm memory for context-evoked drug craving in rats. Neuropsychopharmacology 37: 2101-2108.

Engelmann JM, Versace F, Robinson JD, Minnix JA, Lam CY, Cui Y et al (2012). Neural substrates of smoking cue reactivity: a metaanalysis of fMRI studies. NeuroImage 60: 252-262.

Forget B, Pushparaj A, Le Foll B (2010). Granular insular cortex inactivation as a novel therapeutic strategy for nicotine addiction. Biol Psychiatry 68: 265-271.

Fuchs RA, Evans KA, Ledford CC, Parker MP, Case JM, Mehta RH et al (2005). The role of the dorsomedial prefrontal cortex, basolateral amygdala, and dorsal hippocampus in contextual reinstatement of cocaine seeking in rats. Neuropsychopharmacology 30: 296-309.

Fuchs RA, Lasseter HC, Ramirez DR, Xie X (2008). Relapse to drug seeking following prolonged abstinence: the role of environmental stimuli. Drug Discov Today Dis Models 5: 251-258.

Gaznick N, Tranel D, McNutt A, Bechara A (2014). Basal ganglia plus insula damage yields stronger disruption of smoking addiction than basal ganglia damage alone. Nicotine Tob Res 16: 445-453.

Heatherton TF, Kozlowski LT, Frecker RC, Fagerstrom KO (1991). The Fagerstrom Test for Nicotine Dependence: a revision of the Fagerstrom Tolerance Questionnaire. Br J Addict 86: 1119-1127.

Janes AC, Pizzagalli DA, Richardt S, de BFB, Chuzi S, Pachas G et al (2010). Brain reactivity to smoking cues prior to smoking cessation predicts ability to maintain tobacco abstinence. Biol Psychiatry 67: 722-729.

Jenkinson M, Bannister P, Brady M, Smith S (2002). Improved optimization for the robust and accurate linear registration and motion correction of brain images. NeuroImage 17: 825-841.

Jenkinson M, Beckmann CF, Behrens TE, Woolrich MW, Smith SM (2012). Fsl. NeuroImage 62: 782-790.
Kuhn S, Gallinat J (2011). Common biology of craving across legal and illegal drugs-a quantitative meta-analysis of cue-reactivity brain response. Eur J Neurosci 33: 1318-1326.

Liu Y, Le Foll B, Wang X, Lu L (2008). Conditioned place preference induced by licit drugs: establishment, extinction, and reinstatement. ScientificWorldJournal 8: 1228-1245.

Maldjian JA, Laurienti PJ, Kraft RA, Burdette JH (2003). An automated method for neuroanatomic and cytoarchitectonic atlas-based interrogation of fMRI data sets. NeuroImage 19: 1233.

Marchant NJ, Kaganovsky K, Shaham Y, Bossert JM (2014). Role of corticostriatal circuits in context-induced reinstatement of drug seeking. Brain Res (e-pub ahead of print).

Maren S, Phan KL, Liberzon I (2013). The contextual brain: implications for fear conditioning, extinction and psychopathology. Nat Rev Neurosci 14: 417-428.

Marschner A, Kalisch R, Vervliet B, Vansteenwegen D, Buchel C (2008). Dissociable roles for the hippocampus and the amygdala in human cued versus context fear conditioning. J Neurosci 28: 9030-9036.

McClernon FJ, Hiott FB, Huettel SA, Rose JE (2005). Abstinenceinduced changes in self-report craving correlate with event-related FMRI responses to smoking cues. Neuropsychopharmacology 30: 1940-1947.

McClernon FJ, Hiott FB, Liu J, Salley AN, Behm FM, Rose JE (2007). Selectively reduced responses to smoking cues in amygdala following extinction-based smoking cessation: results of a preliminary functional magnetic resonance imaging study. Addict Biol 12: 503-512.

McClernon FJ, Kozink RV, Lutz AM, Rose JE (2009). 24-h smoking abstinence potentiates fMRI-BOLD activation to smoking cues in cerebral cortex and dorsal striatum. Psychopharmacology (Berl) 204: 25-35.

McClernon FJ, Kozink RV, Rose JE (2008). Individual differences in nicotine dependence, withdrawal symptoms, and sex predict transient fMRI-BOLD responses to smoking cues. Neuropsychopharmacology 33: 2148-2157.

McLaughlin J, See RE (2003). Selective inactivation of the dorsomedial prefrontal cortex and the basolateral amygdala attenuates conditioned-cued reinstatement of extinguished cocaine-seeking behavior in rats. Psychopharmacology (Berl) 168: $57-65$.

Meyers RA, Zavala AR, Neisewander JL (2003). Dorsal, but not ventral, hippocampal lesions disrupt cocaine place conditioning. Neuroreport 14: 2127-2131.

Meyers RA, Zavala AR, Speer CM, Neisewander JL (2006). Dorsal hippocampus inhibition disrupts acquisition and expression, but not consolidation, of cocaine conditioned place preference. Behav Neurosci 120: 401-412.

Napier TC, Herrold AA, de Wit H (2013). Using conditioned place preference to identify relapse prevention medications. Neurosci Biobehav Rev 37: 2081-2086.

Naqvi NH, Gaznick N, Tranel D, Bechara A (2014). The insula: a critical neural substrate for craving and drug seeking under conflict and risk. Ann N Y Acad Sci 1316: 53-70.

Naqvi NH, Rudrauf D, Damasio H, Bechara A (2007). Damage to the insula disrupts addiction to cigarette smoking. Science 315: 531-534.

O'Brien CP, Childress AR, McLellan AT, Ehrman R (1992). Classical conditioning in drug-dependent humans. Ann $N Y$ Acad Sci 654: 400-415.

Otis JM, Fitzgerald MK, Mueller D (2014). Inhibition of hippocampal beta-adrenergic receptors impairs retrieval but not reconsolidation of cocaine-associated memory and prevents subsequent reinstatement. Neuropsychopharmacology 39: 303-310.

Poppenk J, Evensmoen HR, Moscovitch M, Nadel L (2013). Longaxis specialization of the human hippocampus. Trends Cogn Sci 17: 230-240. 
Pushparaj A, Hamani C, Yu W, Shin DS, Kang B, Nobrega JN et al (2013). Electrical stimulation of the insular region attenuates nicotine-taking and nicotine-seeking behaviors. Neuropsychopharmacology 38: 690-698.

Ramirez DR, Bell GH, Lasseter HC, Xie X, Traina SA, Fuchs RA (2009). Dorsal hippocampal regulation of memory reconsolidation processes that facilitate drug contextinduced cocaine-seeking behavior in rats. Eur J Neurosci 30: 901-912.

Stalcup SA, Christian D, Stalcup J, Brown M, Galloway GP (2006). A treatment model for craving identification and management. J Psychoactive Drugs 38: 189-202.
Strange BA, Witter MP, Lein ES, Moser EI (2014). Functional organization of the hippocampal longitudinal axis. Nat Rev Neurosci 15: 655-669.

Tang DW, Fellows LK, Small DM, Dagher A (2012). Food and drug cues activate similar brain regions: a meta-analysis of functional MRI studies. Physiol Behav 106: 317-324.

Wetherill RR, Young KA, Jagannathan K, Shin J, O'Brien CP, Childress AR et al (2013). The impact of sex on brain responses to smoking cues: a perfusion fMRI study. Biol Sex Differ 4: 9.

Wolosin SM, Zeithamova D, Preston AR (2012). Reward modulation of hippocampal subfield activation during successful associative encoding and retrieval. J Cogn Neurosci 24: 1532-1547.

Supplementary Information accompanies the paper on the Neuropsychopharmacology website (http://www.nature.com/npp) 